Three Generalizability Studies of the Components of Perceived Coach Support Please reference this paper as:

Rees, T., Freeman. P., Bell, S., \& Bunney, R. (2012). Three generalizability studies of the components of perceived coach support. Journal of Sport \& Exercise Psychology, 34, 238-251.

Submitted: $23^{\text {rd }}$ January 2012 


\begin{abstract}
Coaches are important providers of social support, but what influences us to perceive our coaches as supportive or unsupportive? We investigated the extent to which perceptions of coach support reflect characteristics of athletes and coaches, as well as relational components. In three studies, athletes judged the actual or hypothetical supportiveness of various coaches. The methods of generalizability theory permitted us to conclude that perceptions of coach support primarily reflected relational components, with characteristics both of athletes and coaches also independently playing (lesser) roles. These findings suggest that athletes may systematically disagree on the supportiveness of their coaches.
\end{abstract}

Key Words: athletes, social support, perceivers, targets, generalizability theory 
Three Generalizability Studies of the Components of Perceived Coach Support

What influences us to perceive one coach as supportive and another as unsupportive? The importance of this question lies in the fact that: (a) coaches play a major role in sport, and social support is integral to the coaching process (Bianco, 2001, Chelladurai \& Saleh, 1980;

Kristiansen, \& Roberts, 2010; Rosenfeld, Richman, \& Hardy, 1989; Robbins \& Rosenfeld, 2001), and (b) perceived support is consistently and strongly related to psychological, physiological, and behavioral outcomes (Cohen, Underwood, \& Gottlieb, 2000), including selfefficacy, self-confidence, and sports performance (e.g., Freeman \& Rees, 2008, 2009; Rees \& Freeman, 2007, 2009); but (c) evidence for the success of social support interventions in improving either perceived support, psychological health, or performance is mixed (Freeman, Rees, \& Hardy, 2009; Hogan, Linden, \& Najarian, 2002). Translating social support research into effective interventions may thus require an understanding of what influences people to perceive (or judge) others as more or less supportive (Lakey \& Lutz, 1996; Hogan et al., 2002). In this research, we report three studies of the components of perceived coach support.

Although variously defined (Veiel \& Baumann, 1992), social support is comprised of three major sub-constructs (Lakey, 2010). Social integration reflects the number of different types of relationships in which recipients participate (e.g., coach, team-mate, friends, family members). Enacted support reflects the specific helping actions provided by coaches, teammates, friends, and family members, usually during a specific time frame. Perceived support refers to one's potential access to social support and is a support recipient's subjective judgment that coaches, team-mates, friends, and family members, would provide assistance if needed. Links between social integration and psychological outcomes have been inconsistent, and enacted support has either been unrelated or even negatively related to psychological outcomes (e.g., Barrera, 1986; Finch, Okun, Pool, \& Ruehlman, 1999). Instead, as we noted above, it is 
individuals who perceive their relationships as supportive who have been shown to experience a range of favorable outcomes.

Although coaches have been noted as particularly key support providers, it is unclear what influences us to perceive our coaches as supportive or unsupportive. Theoretical models of social support have differed in the proposed determinants of support perceptions, with the potential for both characteristics of the recipient and the environment to play a role. Both traditionally and intuitively, it might be assumed that people base their perceptions of support on characteristics of the provider and the amount and quality of the specific supportive actions provided (e.g., emotional, esteem, informational, and tangible forms of enacted support: Rees \& Hardy, 2000). However, it has been noted that perceived and enacted support may share as little as $12 \%$ common variance (Haber, Cohen, Lucas, \& Baltes, 2007) and are now widely considered two key but separate constructs (Dunkel-Schetter \& Bennett, 1990; Helgeson, 1993; Wethington $\&$ Kessler, 1986). Given this evidence, perceptions of support must reflect more than just the support recently enacted.

In attempting to unpack how people generate perceptions of support, recent research has applied methods from Generalizability Theory (Brennan, 2001; Cronbach, Gleser, Nanda, \& Rajaratnam, 1972; Shavelson \& Webb, 1991). When recipients of support rate all providers of support within a given study, these methods allow the examination of the extent to which perceived support reflects the characteristics of the people making the judgments (i.e., perceivers), the characteristics of the people being judged (i.e., the supporters or targets), and a unique perceiver-target relational component (e.g., Lakey, McCabe, Fisicaro, \& Drew, 1996; Lakey \& Scoboria, 2005; and see Lakey, 2010). Perceiver components reflect the extent to which perceivers differ in their support judgments across all targets, regardless of any specific target traits or behaviors. For example, applied to coaching, one athlete might rate the supportiveness 
of all coaches more favorably compared to another athlete. Target components reflect the extent to which some targets are seen by all perceivers as more supportive than other targets. For example, all athletes might rate one coach as more supportive than another coach. Relational components reflect inconsistency in perceivers' support judgments across different targets, in that certain perceivers would rate certain targets as more supportive than other targets. In other words, relational components reflect differences in opinion about who is supportive. For example, an offensive football player might rate the head coach as more supportive than does a defensive player, but the defensive player might rate an assistant coach as more supportive than does the offensive player.

Although this line of research is still in its relative infancy, preliminary estimates from studies in general social psychology suggest that the largest component of perceived support is relational. For example, relational components have accounted for between $21 \%$ and $72 \%$ of the variance in perceived support with students rating professors, university sorority members rating one another, depressed inpatients rating videoed targets, and therapy patients rating therapists (e.g., Lakey et al., 1996; Lakey, Drew, \& Sirl, 1999; Lakey, Cohen, \& Neely, 2008). A recent analysis suggests that when real-world samples and targets are used, relational components may account on average for as much as $62 \%$ of the variance in perceived support (Lakey, 2010). Perceiver components tend to be smaller, accounting on average for $27 \%$ of the variance, while target components account on average for only 7\% (Lakey, 2010). The latter finding may run counter to most people's view of themselves as objectively supportive. That is, some might feel target effects are underestimated. Although the available empirical evidence does not bear out this objection, we believe that judgments of coach support might provide contrary evidence. The coaching process is one in which there is a reasonably well-defined social norm about what 
coaches are supposed to do to be helpful (e.g., see Jowett \& Poczwardowksi, 2007), and thus agreement on target support should be higher in coaching compared with other contexts.

Regardless, the identification of perceiver, target, and/or relational components has important applied implications, because it potentially offers a clear focus for intervention. For example, given the figures above, interventions designed to focus purely on target influences may be misguided - a potential reason for the mixed success of support interventions (Hogan et al., 2002; Lakey \& Lutz, 1996). The identification of significant relational components in the present studies would, however, challenge the appealing notion that some coaches are characteristically and objectively more supportive than others. In fact, it would suggest that athletes may systematically disagree on the supportiveness of coaches. Therefore, the introduction of one apparently supportive new coach to a group of athletes may not be effective for all athletes. Instead, to enhance intervention efficacy, one might consider adopting a strategy of carefully matching athletes with specific coaches.

The goal of the present research was to use Generalizability Theory to examine athletes' perceptions of coach support. In order to isolate perceiver, target, and relational components, all participants are required to rate the same targets. As Lakey, Lutz, and Scoboria (2004) noted, this leads to difficulty in finding naturalistic contexts in which a sufficient number of targets are well known to all participants. With this in mind, we conducted three differing studies: Study 1 was a hypothetical study, in which soccer players rated the level of support they believed would be available to them if they were playing under the proposed soccer managers; In Study 2, university athletes rated the supportiveness of coaches on video; In Study 3, academy athletes rated the real-world supportiveness of their coaches. In each study, each participant rated each target, resulting in a fully crossed design, enabling us to determine the percentage of variance accounted for by perceiver, target, and relational components. As the design for each study was 
essentially identical, rather than discussing the results of each study separately, we present all our interpretations in one overall Discussion. To increase the statistical power to detect target effects, the data from the three studies were also combined into a partially nested design in a fourth analysis. Such an analysis does not inflate variances, but it does decrease the risk of Type 2 errors. In line with previous research in general social psychology, it was hypothesized that relational components would account for the greatest amount of variance in perceptions of coach support.

Study 1

Method

\section{Sample and Procedure}

The study was approved by an institutional ethics committee review, and participants provided informed consent. Fifty male competitive (club-level) soccer players (Mean age = $20.18, S D=1.50)$ were asked to rate the supportiveness of five well-known soccer managers from the English Premier League. The players currently had a coach, and generally trained twice per week and played matches twice per week. The managers $($ Mean age $=54.40, S D=9.56)$ were of different nationalities, possessed a minimum of four years management experience (in the English Premier League), had managed at least two clubs, and had managed their current club for at least two years. Although the managers were not observed to provide any specific supportive actions, it was believed that this method was appropriate for studying how participants rated support. Previous research has successfully adopted similarly novel approaches, such as participants rating the perceived supportiveness of TV characters (Lakey et al., 2004). As Lakey et al. noted, such strategies share common features with real-life, naturalistic contexts, in that participants have had the opportunity to view targets across various 
situations and displaying different forms of behavior. In the present study, all participants reported some $(n=35)$ or a lot of knowledge $(n=15)$ of the managers.

To protect the confidentiality of the managers, we adopted a similar procedure to Lakey et al. (1996). Rather than including the managers' names on the questionnaires, a coding system was used. A research assistant constructed the code and handed a coding booklet to participants along with the questionnaire packets. Participants returned the questionnaires in sealed envelopes, and the research assistant removed the coding and consent forms. This process ensured the investigators were unaware of the support ratings of specific managers and which participants provided the ratings. The research assistant was not involved in any other aspect of Study 1 . The order of the presentation of targets was randomized.

\section{Measures}

Target Supportiveness. Target supportiveness was assessed by way of nine items, adapted from the questionnaire used by Freeman and Rees (2009) to measure perceived support. As Freeman and Rees used high-level golfers and assessed support from all potential providers, we chose items based upon their relevance and applicability for the samples in the present set of studies and the focus on coaches'/managers' support. This followed the recommendation from the social support literature that social support measures should be relevant to the situational context in which they are being used (Bianco \& Eklund, 2001; House \& Kahn, 1985; Wills \& Shinar, 2000). The nine items reflected emotional, esteem, and informational forms of support. Tangible items were deemed inappropriate, in particular for Study 1, in which tangible forms of support (material aid, actual help with tasks) would have been more likely from clubs rather than the coaches/managers. Following the procedure of Freeman and Rees (2009), prior to data collection, the study authors, an independent sport psychology researcher from a separate institution, a group of 10 (5 male; 5 female) postgraduate university students who had completed 
modules in sport psychology and social support, and two university-level coaches scrutinized the items for relevance and representativeness. The judges were required to read each item and indicate (by circling yes or no) the relevance of those support items for the coaching of athletes. All judges agreed to the relevance of all nine items. Additionally, the judges confirmed that the items were representative of the typical supportive behavior of coaches.

Participants were asked to rate how supportive each manager would be to the participant if the participant actually played under that manager. The measure asked respondents, "To what extent do you feel ... [manager's code] . . would . ..," with response options ranging on a 5point scale from 1 (not at all) to 5 (a lot). Sample items included: “Tell you, you can do it?" and “Give you constructive criticism?” Although the support measure did contain emotional, esteem, and informational forms of support, we did not distinguish between the dimensions in our analysis. While social support may be broken down into specific dimensions conceptually, in naturalistic settings the dimensions are not usually independent (Cohen \& Wills, 1985), and researchers often use unidimensional measurement of support. In this study, we followed the practice of previous generalizability research (e.g., Lakey et al., 2004; Neely et al., 2006), for which the focus is on overall support perceptions rather than examination of specific dimensions. Internal consistency for the supportiveness ratings of the five managers using this measure ranged from .79 to .83 .

\section{Statistical Analyses}

Univariate generalizability theory was applied to examine perceiver, target, and relational components of perceived support using a fully crossed design. Variance components and standard errors were computed using restricted maximum likelihood variance estimation, using the variance components procedure in SPSS, version 19. Questionnaire items and targets were within-subjects factors, and perceivers were the between-subjects factor. Each participant was a 
level of the perceivers factor and each manager was a level of the targets factor. To reduce measurement error and simplify the design, the odd and even questionnaire items were combined to form two indicators of support (Lakey et al., 2004), which were levels of the items factor. As Shavelson and Webb (1991) noted, the highest order interaction (perceivers*targets*items) was confounded with unmeasured sources of variation and was therefore used as the error term. For the purpose of this study, we focus on perceiver, target, and relational components, and thus do not report all possible components. All components were, however, used to calculate the total variance. The perceiver, target, and relational components were significant when their 95\% confidence intervals did not include 0 .

\section{Results}

The variance components, $95 \%$ confidence intervals, and percentages of variance accounted for by each of the perceiver, target, and relational components are presented in Table 1 for each study. By far the largest contributor to support perceptions was the relational component, accounting for a significant $38 \%$ of the variance. The next largest was the perceiver component, accounting for a significant $21 \%$ of the variance. The target component accounted for $10 \%$ of the variance, although this value was non-significant.

Study 2

Method

\section{Sample and Procedure}

The study was approved by an institutional ethics committee review, and participants provided informed consent. Sixty-nine university athletes $($ Mean age $=19.84, S D=.83 ; 40$ males; 29 females) were asked to rate the supportiveness of five coaches (Mean age $=37.80, S D$ $=10.97 ; 3$ males; 2 females $)$ using videoed targets. Participants competed in a variety of team $(n$ $=43)$ and individual $(n=26)$ sports and all currently had a coach. The performance level of the 
participants comprised club $(n=35)$, county $(n=19)$, national $(n=11)$, and international $(n=4)$ standard. All the participants reported no prior knowledge of the coaches. The coaches possessed a minimum of eight years coaching experience $($ Mean $=15.60, S D=6.19)$ and coached in team $(n=3)$ and individual $(n=2)$ sports. The videos consisted of five 2-minute video clips in which each of the five coaches were seated in an interview suite and asked to talk about his/her style of coaching and the benefits for athlete development of that style. Participants rated the supportiveness of the coaches using the same measure as in Study 1. Questionnaire packets were also prepared and administered in the same way as in Study 1. Internal consistency for the supportiveness ratings of the five coaches ranged from .86 to .93 .

Results

As in Study 1, the largest contributor to support perceptions was the relational component, accounting for a significant $41 \%$ of the variance (see Table 1 ). The perceiver component accounted for a significant $20 \%$ of the variance. The target component accounted for $26 \%$ of the variance, although this value was non-significant ${ }^{1}$.

Study 3

Method

\section{Sample and Procedure}

The study was approved by an institutional ethics committee review, and participants, their parents, and the coaches provided informed consent. Fifty-one youth athletes (Mean age= 13.08, $S D=.87 ; 44$ males, 7 females) enrolled on a "gifted and talented" (identified by coaches as individuals who have achieved at a level significantly in advance of the average for their year group) program were asked to rate the supportiveness of five of their coaches (Mean age = $35.20, S D=5.12 ; 3$ males; 2 females $)$. Participants competed in a variety of team $(n=40)$ and individual $(n=11)$ sports. The performance level of the participants comprised county $(n=46)$ 
and national $(n=5)$ standard. All participants had worked with all the coaches before and reported themselves to have a little $(n=23)$ or detailed knowledge $(n=28)$ of the coaches. The coaches possessed a minimum of 10 years coaching experience $($ Mean $=14.60, S D=4.98)$ and coached in team $(n=4)$ and individual $(n=1)$ sports. Participants rated the supportiveness of the coaches using the same measure as in Studies 1 and 2, and the questionnaire packets were prepared and administered in the same way. Internal consistency for the supportiveness ratings of the five coaches ranged from .94 to .97 .

\section{Results}

As in Studies 1 and 2, the largest contributor to support perceptions was the relational component, accounting for a significant $44 \%$ of the variance. The perceiver component accounted for a significant $22 \%$ of the variance. The target component accounted for $29 \%$ of the variance, although this value was non-significant ${ }^{2}$.

Combined Study Analysis

Method Sample, Procedures, and Statistical Analysis

To increase the statistical power to detect target effects, the data from the three studies were combined into a fourth analysis. The combined sample was therefore 170 participants (Mean age $=17.91, S D=3.35 ; 134$ males, 36 females), each of whom rated 5 out of the 15 possible targets. Participants competed in a variety of team $(n=133)$ and individual $(n=37)$ sports. The performance level of the participants comprised club $(n=85)$, county $(n=65)$, national $(n=16)$, and international $(n=4)$ standard. Data were analyzed as a partially nested design. Items and targets were within-subjects factors; perceivers and study were betweensubjects factors. Perceivers and targets were nested within studies. 
The variance components, $95 \%$ confidence intervals, and percentages of variance accounted for by each component in the combined analysis are presented in Table 1. All three components accounted for significant amounts of variance in support perceptions: The largest was the relational component, accounting for $35 \%$ of the variance ${ }^{3}$; The target and perceiver components accounted for $21 \%$ and $18 \%$ of the variance respectively.

\section{Discussion}

The primary purpose of this research was to examine perceiver, target, and relational components of perceived coach support. If support perceptions were to reflect a stable, trait-like characteristic of perceivers, it would be expected that, compared to other athletes, certain athletes would rate all coaches as more or less supportive. If support perceptions were to reflect characteristics of the targets, it would be expected that all athletes might fundamentally agree on the supportiveness or otherwise of each of the coaches. Finally, if support perceptions were primarily relational, it would be expected that certain athletes would rate certain coaches as more or less supportive. The data from the present studies demonstrate that all three sources of variance made significant contributions to perceptions of coach support, with the primary contributor being the relational component.

The explained variances of $35-44 \%$ for relational components in the present studies were consistent with judgments of support in other contexts (e.g., Lakey et al., 1996; Lakey et al., 1999; Lakey et al., 2008). The present studies of coach support thus add to the understanding of the components of perceived support. Perceived support has traditionally been considered to reflect either support recently received from objectively supportive providers or a stable, traitlike characteristic of the perceiver. As Lakey and Scoboria (2005) noted, rather than these views being competing, they may merely reflect explanations at different levels of analysis. The findings of the present studies suggest that both perceiver and target components may play a role 
in judging the supportiveness of coaches, but that the largest contributor to support perceptions is the relational component. This implies that in rating supportiveness in the present studies, although some coaches/managers were viewed as particularly supportive by certain athletes, the same (apparently supportive) coaches were viewed in a different (and less supportive) light by other athletes. For example, one offensive coordinator might be rated very differently on support by a quarterback than by a lineman. Relational components are the least well-researched source of support variance, but their relative influence suggests traditional views of support perceptions may be limited. That is, perceived support appears not to reflect characteristics of just the athlete or coach. Rather, relational components suggest that athletes are likely to disagree on the supportiveness of the same coaches.

Although the relational components accounted for the greatest amount of variance in support perceptions, perceiver components were also significant, accounting for between $20 \%$ and $22 \%$ of the variance in the three studies and $18 \%$ in the combined analysis. Despite these lower effect sizes, perceiver components should not be ignored. Indeed, these results suggest partial support for the notion that support perceptions may reflect perceiver characteristics, which are stable across different providers of support. From this viewpoint, perceived support could be considered less a reflection of targets, but more a reflection of athletes' tendency to rate all coaches as more or less supportive. For example, soccer player A might rate the supportiveness of all coaches more favorably than does soccer player B. The initial, nonsignificant target effects across the three studies (10-29\% of the variance in support perceptions) might have suggested that there was little inter-participant agreement regarding whether one coach was more supportive than another. However, in the combined analysis, the target variance of $21 \%$ was significant. This points to the fact that studies with just five targets may be insufficiently powerful to detect target effects and may lead to Type 2 errors. It would be 
unusual, however, for studies to be realistically conducted in which athletes are able to reflect on the support of more than five coaches. It is of particular note that the amount of variance accounted for by target components in the present studies is greater than those observed in other contexts. In the general social psychology literature target effects are typically small, leading to claims that target effects are relatively unimportant and/or that there are no objectively supportive features of providers ${ }^{4}$. For example, target components have been found to account for less than $10 \%$ of the variance in the support perceptions of university freshmen rating psychology majors (Veenstra et al., 2011), university sorority members rating one another (Lakey et al., 1996), family members rating each other (Branje, van Aken, \& van Lieshout, 2002), and medical fellows rating clinical faculty (Giblin \& Lakey, 2010). In contrast, as we noted in our introduction, there may be something unique about sport and/or coaching that leads to the higher values we observed in the present studies. In particular, because the role of coaches is relatively well specified in sport, with a well-defined social norm about what coaches are supposed to do to be helpful (e.g., provide training and instruction, guidance, positive feedback, and support: Jowett \& Poczwardowski, 2007; Riemer, 2007), it seems perfectly reasonable that agreement on level of target support should be much higher in the present studies compared with studies using different populations.

The results of the present studies have important implications for social support interventions. Given the significant perceiver components, a perceiver approach would be legitimate, and may involve trying to change the maladaptive support cognitions of an athlete with low perceived support-e.g., challenging the belief that using support is a sign of weakness (Hardy, Jones, \& Gould, 1996). However, perceiver effects reflect the aspect of support that is stable across support providers and time, and thus might not be amenable to change. Furthermore, on average, perceiver components accounted for only $18 \%$ of the variance in 
perceptions of support, so a perceiver-based approach would likely be less effective than a relational-based strategy for increasing support perceptions. Although target components were non-significant in the three studies, the significant effect in the combined analysis potentially provides a challenge to the notion from general social psychology (e.g., see Lakey, 2010) that there may be no objectively supportive features of providers. In light of this result, a target approach could also be legitimate, and might involve the introduction of one objectively supportive coach. It should, however, be noted that evidence for the success of support interventions that have introduced one provider has been mixed (Hogan et al., 2002). Attempting to introduce one objectively supportive coach may simply not be practical, and it might ignore other important qualities of coaching, such as experience, skill-level, trust, respect, communication skills, and understanding (Jowett \& Poczwardowski, 2007). Given that the relational component accounted for the greatest amount of variance in support perceptions, a relational approach may still be the most promising focus of interventions. For this approach, athletes would need to be matched with specific coaches, to ensure a good fit. For example, similarity in attitudes, experiences, or personalities could be used to match athletes and coaches. Although a matching approach offers the best potential for ensuring perceptions of coach support are maximized, one could see that this could have the potential to become a time-consuming task. However, preliminary evidence (Veenstra et al., 2011) suggests that support recipients can accurately forecast later relational support even following brief conversations with potential support providers. For example, using university freshmen interacting with psychology majors, Veenstra et al. (Study 1) successfully forecasted relational support (i.e., successfully matched perceivers with providers) over a three-week period, based on recipients' positive affect and judgments of providers' supportiveness following an initial 10-minute conversation. Veenstra et al. (Study 2) then replicated this effect in a mixed sample of students and workers over a four- 
month period. It may not, however, be possible to employ such a matching strategy in all sports. For example, some teams might only have one coach, thereby eliminating the potential to match athletes with coaches. In contrast, in individual sports such as athletics, tennis, and golf, athletes might have greater freedom to opt for (or be matched with) a particular coach.

Although the basis on which to conduct matching will be an important avenue for future research, the question remains, what factors or mechanisms lead to the effects we observed in the present set of studies? For example, why might some athletes have a tendency to report all coaches as more supportive compared to other athletes (perceiver effect)? Similarly, why might athletes agree that some coaches are more supportive (target effect)? And why might some athletes disagree on the supportiveness of certain coaches (relational effect)? Perceiver effects might be due to perceptual biases of the perceivers (Lakey \& Drew, 1997). For example, Lakey, Moineau, and Drew (1992) found that individuals with characteristically low perceived support rated the supportiveness of videotaped support attempts less favorably than individuals with characteristically high perceived support. Certain personality characteristics such as agreeableness (Lakey et al., 2004) have been associated with target effects. Relational effects may result from perceivers using different information to rate support providers, such that in rating supportiveness, one athlete may draw upon the perceived similarity of coaches to themselves, whereas another athlete might draw upon coaches' personality traits. Lutz and Lakey (2001) found that perceivers' personality predicted the extent to which they drew upon different traits to rate the supportiveness of providers. The implication of this work is that one athlete might view a coach as supportive if the coach were to exhibit openness, whereas another athlete might view a coach as supportive if the coach were to exhibit agreeableness. Lutz and Lakey even found that, compared to perceivers low in neuroticism, perceivers high in neuroticism saw targets high in neuroticism as more supportive. The latter finding might be influenced by 
people's preferences for how support-related conversations should be carried out: A support provider low in neuroticism might not meet the high neuroticism perceiver's preference for or expectation of support; conversely, the support provider high in neuroticism might show more empathy for the negative emotion of the high neuroticism perceiver.

Having identified that perceiver, target, and relational components underpin perceived coach support, future research should also examine the unique effects of the different components (Lakey, 2010; Lutz \& Lakey, 2001). That is, just because the relational component was the largest contributor to perceived coach support does not mean it is related to key outcomes of interest, such as emotions, self-efficacy, and performance. Ultimately, we would want evidence that an athlete who sees a coach as especially supportive performs particularly well in the presence of that coach. To examine such effects requires studies in which participants are assessed on relevant criterion variables in the presence of each coach/support provider. Multivariate generalizability analyses may then be used to determine the correlations between support perceptions and the criterion variables at the various component levels.

Some potential limitations of the present studies should be noted. First, a limitation of Studies 1 and 2 is that participants did not rate members of their own network, which may limit the ecological validity of these studies. The results were, however, congruent with those found in Study 3, in which participants did rate members of their own network. Second, one could also argue that with regard to Study 3, although participants rated members of their own network, by restricting participants to all rate the same five coaches, we may have excluded some participants' most supportive coach. Future research could ask participants to rate their most important coaches (or indeed other support providers). This was the strategy employed by Barry, Lakey, and Orehek (2007) and Lakey and Scoboria (2005), in whose studies participants rated their own important providers of support. The nature of these designs means, however, that 
providers (targets) are then nested within perceivers, such that provider and relational components cannot be distinguished. Third, in Studies 2 and 3 athletes and coaches were drawn from individual and team sports. If individual-sport athletes were to have found it difficult to rate team-sport coaches (and vice versa), this might have led to greater disagreement among athletes, thereby inflating the relational effect. The results were nonetheless very similar to Study 1 , in which the focus was explicitly on the team sport of soccer. Finally, one could argue that in Study 2 , instead of allowing coaches to speak freely, advice from coaching experts might have been sought to manipulate the content of the videos. The focus of all of the studies was, however, on athletes' naturally occurring perceptions of coach support. It would not, therefore, have been relevant or appropriate to attempt to manipulate the style or content of the coaching videos.

In conclusion, the present studies help inform our understanding of perceived coach support by demonstrating the relative contributions of perceiver, target, and relational components. Although support perceptions reflected all three components, the largest contributor was the relational component. Thus the answer to the question: "What influences us to perceive one coach as supportive and another as unsupportive?" would be, "it depends." Researchers and applied practitioners may need to consider the match between athletes and coaches to ensure supportive relationships are formed. Attention to the different components will help develop understanding of how support perceptions are formed, relationships between support and outcomes, and the most appropriate focus for social support interventions. 


\section{Footnotes}

${ }^{1}$ To examine if gender of the perceiver moderated the amount of variance accounted for by perceiver, target, and relational components, the analysis was repeated for male and female athletes separately. A significant gender difference would be apparent if it could be demonstrated that the $95 \%$ confidence intervals for the two samples did not overlap. The $95 \%$ confidence intervals did, however, overlap for perceiver, target, and relational components, thus demonstrating that these effects did not differ for males and females. To examine if gender of the target influenced the amount of variance accounted for by support perceptions, analysis was repeated with target nested within gender. The $95 \%$ confidence intervals for each of the perceiver, target, and relational components overlapped in the nested and original analyses, indicating that these effects did not differ across male and female targets. In addition, target gender did not account for a significant amount of variance in support perceptions.

${ }^{2}$ Similar to the additional analyses (see Footnote 1) in Study 2, there were no effects for gender of perceivers or targets.

${ }^{3}$ Note the magnitude of variance accounted for by the relational components has been reduced compared to the individual studies. This is due to inclusion of the study factor, which accounted for a non-significant $16 \%$ of the variance in perceptions of coach support.

${ }^{4}$ As Lakey (2010) pointed out, when all participants rate the same targets, target components reflect the extent to which targets differ in their rated supportiveness, averaged across participants' ratings. To the extent that inter-rater agreement may be considered an index of objective reality, target components reflect the extent to which supportiveness is an objective feature of targets. 


\section{References}

Barrera, M. J. (1986). Distinctions between social support concepts, measures, and models. American Journal of Community Psychology, 14, 413-445.

Barry, R., Lakey, B., \& Orehek, E. (2007). Links among attachment dimensions, affect and the self for broadly-generalized attachment styles and relationship-specific bonds. Personality and Social Psychology Bulletin, 33, 240-253.

Bianco, T. (2001). Social support and recovery from sport injury: Elite skiers share their experiences. Research Quarterly For Exercise and Sport, 72, 376-388.

Bianco, T., \& Eklund, R. C. (2001). Conceptual considerations for social support research in sport and exercise settings: The case of sport injury. Journal of Sport \& Exercise Psychology, 23, 85-107.

Branje, S. J. T., van Aken, M. A. G., \& van Lieshout, C. F. M. (2002). Relational support in families with adolescents. Journal of Family Psychology, 16, 351-362.

Brennan, R. L. (2001). Generalizability theory. New York: Springer.

Chelladurai, P. \& Saleh, S. D. (1980). Dimensions of leader behavior in sports: Development of a leadership scale. Journal of Sport Psychology, 2, 34-45.

Cohen, S., Underwood, L. G., \& Gottlieb, B. H. (2000). Social support measurement and intervention: A guide for health and social scientists. New York: Oxford University Press.

Cohen, S., \& Wills, T. A. (1985). Stress, social support and the buffering hypothesis. Psychological Bulletin, 98, 310-357.

Cronbach, L. J., Gleser, G. C., Nanda, H., \& Rajaratnam, N. (1972). The dependability of behavioral measurements: Theory of generalizability for scores and profiles. New York: 
Wiley.

Dunkel-Schetter, C., \& Bennett, T. L. (1990). Differentiating the cognitive and behavioral aspects of social support. In B. R. Sarason, I. G. Sarason \& G. R. Pierce (Eds.), Social support: An interactional view (pp. 267-296). New York: Wiley.

Finch, J. F., Okun, M. A., Pool, G. J., \& Ruehlman, L. S. (1999). A comparison of the influence of conflictual and supportive social interactions on psychological distress. Journal of Personality, 67, 581-622.

Freeman, P., \& Rees, T. (2008). The effects of perceived and received support on objective performance outcome. European Journal of Sport Sciences, 8, 359-368.

Freeman, P., \& Rees, T. (2009). How does perceived support lead to better performance? An examination of potential mechanisms. Journal of Applied Sport Psychology, 21, 429-441.

Freeman, P., Rees, T., \& Hardy, L. (2009). An intervention to increase social support and improve performance. Journal of Applied Sport Psychology, 21, 186-200.

Giblin, F., \& Lakey, B. (2010). Integrating mentoring and social support research within the context of stressful medical training. Journal of Social and Clinical Psychology, 29, $771-796$.

Haber, M. G., Cohen, J. L., Lucas, T., \& Baltes, B. B. (2007). The relationship between selfreported received and perceived social support: A meta-analytic review. American Journal of Community Psychology, 39, 133-144

Hardy, L., Jones, G., \& Gould, D. (1996). Understanding psychological preparation for sport: Theory and practice of elite performers. Chichester, UK: Wiley.

Helgeson, V. S. (1993). Two important distinctions in social support: Kind of support and perceived versus received. Journal of Applied Social Psychology, 23, 825-845. 
Hogan, B. E., Linden, W., \& Najarian, B. (2002). Social support interventions: Do they work? Clinical Psychology Review, 22, 381-440.

House, J. S., \& Kahn, R. L. (1985). Measures and concepts of social support. In S. Cohen \& S. L. Syme (Eds.), Social support and health (pp. 83-108). New York: Academic.

Jowett, S., \& Poczwardowksi, A. (2007). Understanding the coach-athlete relationship. In S. Jowett \& D. Lavallee (Eds.), Social psychology in sport (pp. 3-14). Champaign, IL: Human Kinetics.

Kristiansen, E., \& Roberts, G. C. (2010). Young elite athletes and social support: Coping with competitive and organizational stress in "Olympic" competitions. Scandinavian Journal of Medicine and Science in Sports, 20, 686-695.

Lakey, B. (2010). Social support: Basic research and new strategies for intervention. In J. E. Maddux \& J. P. Tangney (Eds.), Social psychological foundations of clinical psychology (pp. 177-194). New York: Guilford.

Lakey, B., Cohen, J. L. \& Neely, L. C. (2008). Perceived support and relational effects in psychotherapy process constructs. Journal of Counseling Psychology, 55, 209-220.

Lakey, B. \& Drew, J. B. (1997). A social-cognitive perspective of social support. In G. R. Pierce, B. Lakey, I. G. Sarason, \& B. R. Sarason (Eds.), Sourcebook of social support and personality (pp. 107-140). NY: Plenum.

Lakey, B., Drew, J. B., \& Sirl, K. (1999). Clinical depression and perceptions of supportive others: A generalizability analysis. Cognitive Therapy and Research, 23, 511-533.

Lakey, B., \& Lutz, C. J. (1996). Increasing social support: preventive and therapeutic interventions. In G. R. Pierce, B. R. Sarason, \& I. G. Sarason (Eds.), Handbook of social support and the family (pp. 435-466). NY: Plenum. 
Lakey, B., Lutz, C. J., \& Scoboria, A. (2004). The information used to judge supportiveness depends on whether the judgment reflects the personality of perceivers, the objective characteristics of targets, or their unique relationship. Journal of Social and Clinical Psychology, 23, 796-814.

Lakey, B., McCabe, K., Fisicaro, S., \& Drew, J. (1996). Personal and environmental determinants of social support: Three generalizability studies. Journal of Personality and Social Psychology, 70, 1270-1280.

Lakey B., Moineau, S., \& Drew, J. B. (1992). Perceived social support and individual differences in the interpretation and recall of supportive behavior. Journal of Social and Clinical Psychology, 11, 336-348.

Lakey, B., \& Scoboria, A. (2005). Trait and social influences in the links among perceived social support, affect and self esteem. Journal of Personality, 73, 361-388.

Lutz, C. J., \& Lakey, B. (2001). How people make support judgments: Individual differences in the traits used to infer supportiveness in others. Journal of Personality and Social Psychology, 81, 1070-1079.

Rees, T., \& Freeman, P. (2007). The differential impact of perceived and received support upon confidence. Journal of Sports Sciences, 25, 1057-1065.

Rees, T., \& Freeman, P. (2009). Social support moderates the relationship between stressors and task performance through self-efficacy. Journal of Social and Clinical Psychology, 28, 245-264.

Rees, T., \& Hardy, L. (2000). An investigation of the social support experiences of high-level sport performers. The Sport Psychologist, 14, 327-347. 
Riemer, H. A. (2007). Multidimensional model of coach leadership. In S. Jowett \& D. Lavallee (Eds.), Social psychology in sport (pp. 57-73). Champaign, IL: Human Kinetics.

Robbins, J. E., \& Rosenfeld, L. B. (2001). Athletes' perceptions of social support provided by their head coach, assistant coach, and athletic trainer, pre-injury and during rehabilitation. Journal of Sport Behavior, 24, 277-297.

Rosenfeld, L. B., Richman, J. M., \& Hardy, C. J. (1989). Examining social support networks among athletes: Description and relationship to stress. The Sport Psychologist, 3, 23-33.

Shavelson, R. J., \& Webb, N. M. (1991). Generalizability theory: A primer. Newbury Park: CA: Sage.

Veenstra, A. L., Lakey, B., Cohen, J. L., Neely, L. C., Orehek, E., Barry, R., \& Abeare, C. A. (2011). Forecasting the specific providers that recipients will perceive as unusually supportive. Personal Relationships.

Veiel, H. O. F., \& Baumann, U. (1992). The meaning and measurement of social support. New York: Hemisphere.

Wethington, E., \& Kessler, R. C. (1986). Perceived support, Received support, and adjustment to stressful life events. Journal of Health and Social Behavior, 27, 78-89.

Wills, T. A., \& Shinar, O. (2000). Measuring perceived and received social support. In S. Cohen, L. G. Underwood, \& B. H. Gottlieb (Eds.), Social support measurement and intervention: A guide for health and social scientists (pp. 86-135). New York: Oxford University Press. 
Table 1

Variance Components, 95\% Confidence Intervals, and Percentages of Variance Accounted for by Each Component for Each Study and in the Combined Analysis.

\begin{tabular}{|c|c|c|c|}
\hline Component & Variance components & $95 \%$ confidence interval & $\%$ of variance \\
\hline \multicolumn{4}{|c|}{ Study 1} \\
\hline Perceivers & $.09 *$ & $.03-.15$ & 21 \\
\hline Targets & .04 & $-.02-.11$ & 10 \\
\hline Relational & $.17^{*}$ & $.17-.17$ & 38 \\
\hline \multicolumn{4}{|c|}{ Study 2} \\
\hline Perceivers & $.11^{*}$ & $.05-.17$ & 20 \\
\hline Targets & .15 & $-.05-.35$ & 26 \\
\hline Relational & $.23 *$ & $.23-.23$ & 41 \\
\hline \multicolumn{4}{|c|}{ Study 3} \\
\hline Perceivers & $.27 *$ & $.12-.42$ & 22 \\
\hline Targets & .36 & $-.15-.88$ & 29 \\
\hline Relational & $.54^{*}$ & $.43-.65$ & 44 \\
\hline \multicolumn{4}{|c|}{ Combined Analysis } \\
\hline Perceivers & $.15^{*}$ & $.10-.20$ & 18 \\
\hline Targets & $.18^{*}$ & $.03-.34$ & 21 \\
\hline Relational & $.31 *$ & $.27-.34$ & 35 \\
\hline
\end{tabular}

Note: *denotes $p<.05$. 